\title{
Seismic Analysis of an Offshore Structure in Persian Gulf Utilizing a Physical Model
}

\author{
Farhad Hosseinlou $^{1 *}$, Hamid Hokmabady², Alireza Mojtahedi ${ }^{3}$, Samira Mohammadyzadeh ${ }^{4}$ \\ ${ }^{1}$ Graduated Ph.D. Student, Faculty of Civil Engineering, Department of Water Resources Engineering, University \\ of Tabriz; f.hosseinlou@tabrizu.ac.ir \\ ${ }^{2}$ Ph.D. Candidate, Faculty of Civil Engineering, Department of Water Resources Engineering, University of Tabriz, \\ Tabriz, Iran; h.hokmabady@tabrizu.ac.ir \\ ${ }^{3}$ Associated Professor, Faculty of Civil Engineering, Department of Water Resources Engineering, University of \\ Tabriz,Tabriz,Iran; a.mojtahed@tabrizu.ac.ir \\ ${ }^{4}$ Graduated MS Student, Faculty of Civil Engineering, Department of Structural Engineering, University of Tabriz, \\ Tabriz, Iran; s.mohammadyzadeh@gmail.com
}

\section{ARTICLE INFO}

\section{Article History:}

Received: 5 Jul. 2018

Accepted: 6 Mar. 2019

\section{Keywords:}

Fixed offshore jacket platform

Physical Model

Simplified platform model

Seismic Analysis

Model updating

\begin{abstract}
A simple dynamic model of an offshore jacket platform is developed based on the scaled hydro-elastic model of the jacket to estimate the dynamic response of the system. The finite element model of the platform is updated numerically by using the experimental modal analysis (EMA) results. Dynamic characteristics of the improved simple dynamic model (SPM) and idealized model are specified based on updated model properties. The effects of the experimental test are studied to investigate the dynamic response of a scaled model of an offshore jacket platform through the SPM and idealized models. Seismic response of the jacket platform is studied by using the idealized model under an earthquake acceleration. The effects of marine growth and the corrosion are considered within the calculation process by considering the jacket mass and stiffness variation. The developed SPM and idealized model provide a feasible and effective approach for evaluating the dynamic response of the offshore jacket platform. The results indicate the importance of the experimental studies in validating the numerical results and reducing the uncertainties for the fixed marine structures.
\end{abstract}

\section{Introduction}

Over the past decades, jacket-type structures showed great promise in offshore oil and gas industries. Seismic performance of these structures, which is based on their numerical analysis, not only requires a realistic nonlinear structural model, but also needs uncertainties consideration. Uncertainties can be categorized to three distinct factors related to the computer model: (i) inaccuracy in mathematical equations solving method (numerical uncertainty), (ii) imprecision in model parameters definition (parameter uncertainty), and (iii) inexactness and incompleteness in engineering principles modeling, (structural uncertainty) [1]. Any computer based engineering problem is a mixture of mentioned three factors which no distinguished boundary exists among them [1]. Lack of data of the may cause high structural uncertainties which can reduce the structure effectiveness to endure extreme environmental loadings. For this reason, considering precise numerical models are vital for seismic analysis. This fact is due to importance of the system specification in real dynamic response of system under wave and earthquake. In practical cases, verified models should be simultaneously gain based on numerical and experimental approaches. High complexity in marine structures may cause a significant discrepancy between the numerical and experimental data [1]. Discrepancy reduction can be performed through numerical model updating which is one of the most demanded applications in laboratory route and model vibration testing process of the structures [2]. In past few decades, several updating algorithms are employed to improve analytical FE models by utilizing the limited measurement of experimental models. Numerical modeling can be done by using dynamic matrices due to their capability in revealing the physical meanings of the real structure. Model updating in structural dynamics may be classified into two main approaches as: (i) The direct [3], and (ii) The iterative [4]. Among these approached, the iterative approach is 
able to maintain the initial correspondence between the degrees of freedom (DOFs) within the real structure dynamic matrices. The Eigen sensitivity procedure such as the penalty function technique is one type of sensitivity-based iterative updating approach which can be effectively used to minimize computational costs [5].

The FE models of many practical engineering systems are usually very complex with numerous DOFs and measuring all the modal information and also considering all system DOFs experimentally is not possible. In order to solve the DOFs mismatch between the FE model and the experimental model, model reduction schemes should be applied [6,7]. On the other hand, due to importance of environmental loads in the design of fixed marine structures in various stages of manufacturing, transportation, installation and operation $[8,9]$, for the jacket structures in shallow water and in cases that the dominant wave period is obviously higher than the natural period of the structure, wave loads can be applied statically to the structural system $[9,10]$. However, precise results would be gained by employing non-linear time history analysis especially for deep water offshore structures under extreme loading conditions and also by taking the loading randomness into account $[10,11]$. Seismic behavior of the offshore jacket platforms is discussed previously in the literature under wave-iceberg loading, wave-current loading, earthquake loading and wind loading by using different procedures $[12,13,14$, $15,16]$.

In this manuscript, a vibration based iterative model updating methodology via the penalty function based process is performed for seismic analysis of an offshore structure by using experimental data. For this purpose, an improved reduction technique associated with the model updating process is utilized. The experimental test is done by employing a reduced scale hydro-elastic model of an offshore jacket platform (SPD9-Locatted in Persian Gulf). With the wide-spread utilization of the FE software [16, 17], the focus of this study is set on $3 \mathrm{D}$ modeling of the dynamic response of the fixed platforms which is performed for the first time by considering a simplified platform model (SPM). In this manuscript, the effects of an experimental test are studied to investigate the dynamic response of a scaled model of an offshore jacket platform via development of a SPM and idealized model. The effects of marine growth and the corrosion or varying the mass and stiffness of the jacket platform are considered within the calculation process. The developed SPM and idealized model provide a feasible and effective approach for evaluating the dynamic response of the offshore jacket platform. The results denote the importance of experimental studies in numerical result validation and the uncertainty reduction for the fixed marine structures.

\section{Mathematical Background}

However, the FE modeling of offshore jacket structures can be implemented considering all DOFs, mode shapes, and frequencies, the computational time would be far more time-consuming and expensive. To acquire reasonable approximations of dynamic response of system through FE modeling, the optimum approach is to reduce the number of structural DOFs. The DOF reduction process is faced two major limitations: (i) Importance of lower-value frequencies and corresponding mode shapes in describing structural behavior, and (ii) Predictable range of frequencies that the structure experienced as it is subjected to timedependent forcing functions. Just the natural frequencies around the range are of concern in examining resonance possibilities [18]. Based on the full scale model, some certain nodal freedoms are known as master freedoms and the rest are determined slave freedoms, which their mass, stiffness and, loadings would be summarized into the main master freedoms within the dynamic analysis. The main mass and stiffness matrices, considering both master and slave DOFs, of the system can be expressed as follows:

$$
\begin{aligned}
& {\left[\begin{array}{ll}
{\left[M_{m m}\right]} & {\left[M_{m s}\right]} \\
{\left[M_{s m}\right]} & {\left[M_{s s}\right]}
\end{array}\right]} \\
& {\left[\begin{array}{ll}
{\left[K_{m m}\right]} & {\left[K_{m s}\right]} \\
{\left[K_{s m}\right]} & {\left[K_{s s}\right]}
\end{array}\right]}
\end{aligned}
$$

where $m$ and $s$ are the master and the slave coordinates, respectively. By using the above mentioned mass and stiffness matrices and cross coupling terms, Eq. (3) is derived. By removing the slave DOFs, Eq. (3) can be rewritten as Eq. (4):

$$
\begin{aligned}
& {\left[\begin{array}{ll}
{\left[M_{m m}\right]} & {\left[M_{m s}\right]} \\
{\left[M_{s m}\right]} & {\left[M_{s s}\right]}
\end{array}\right]\left\{\begin{array}{l}
\ddot{X}_{m} \\
\ddot{X}_{s}
\end{array}\right\}+} \\
& {\left[\begin{array}{ll}
{\left[K_{m m}\right]} & {\left[K_{m s}\right]} \\
{\left[K_{s m}\right]} & {\left[K_{s s}\right]}
\end{array}\right]\left\{\begin{array}{l}
X_{m} \\
X_{s}
\end{array}\right\}=\left\{\begin{array}{l}
0 \\
0
\end{array}\right\}} \\
& \left\{\begin{array}{l}
X_{m} \\
X_{s}
\end{array}\right\}=\left[\begin{array}{c}
{[I]} \\
-\left[K_{s s}\right]^{-1}\left[K_{s m}\right]
\end{array}\right]\left\{X_{m}\right\}= \\
& {\left[T_{s}\right]\left\{X_{m}\right\}} \\
& {\left[M_{R}\right]=\left[T_{s}^{T}\right][M]\left[T_{s}\right]} \\
& {\left[K_{R}\right]=\left[T_{s}^{T}\right][K]\left[T_{s}\right]}
\end{aligned}
$$

where $T_{s}$ and $I$ denote Guyan transformation and the identify matrix; and also $\left[M_{R}\right]$ and $\left[K_{R}\right]$ stand for the reduced Guyan mass and stiffness matrices, respectively. One of the best practical process for solving large dynamic problems is the improved reduction technique, which is introduced by O'Callahan in 1989. This scheme is an improvement over the Guyan static reduction algorithm by offering a 
term including the inertial effects as pseudo static forces. To reduce the mass and stiffness matrices, a transformation matrix $T_{i}$ is defined as follows:

$$
\begin{aligned}
& {\left[T_{i}\right]=\left[T_{s}\right]+[S][M]\left[T_{s}\right]\left[M_{R}^{-1}\right]\left[K_{R}\right]} \\
& S=\left[\begin{array}{ll}
{[0]} & {[0]} \\
{[0]} & {\left[K_{S S}^{-1}\right.}
\end{array}\right]
\end{aligned}
$$

and finally the new reduced matrices can be derived as Eq. (9) and (10). The rows and columns corresponding to the slave coordinates are disregarded from the mass and stiffness matrices once at a time which allows matrices to adapt to the removal of a slave, and possibly alter the removed DOF.

$$
\begin{aligned}
& {\left[M_{I R S}\right]=\left[T_{i}^{T}\right][M]\left[T_{i}\right]} \\
& {\left[K_{I R S}\right]=\left[T_{i}^{T}\right][K]\left[T_{i}\right]}
\end{aligned}
$$

In this manuscript, the SPM model is employed for the dynamic response analysis as the jacket platform is subjected to wave loading. Details about the SPM and the idealized model including the lumped mass and stiffness labels are shown in Fig.1. Considering the dynamic response and behavior of an offshore jacket platform subjected to wave and current is necessary in the analysis procedure. So, the SPM, which is introduced by Marine Technology and Management group [19], is applied for the dynamic response analysis as the jacket platform is subjected to wave loading. The structural elements are modeled as vertical equivalent cylinders in the direction of the wave crest and the stiffness properties of are considered equivalent to the vertical bracings, legs, and piles stiffness of the initial model. The lateral stiffness of bracing members $\left(K_{b}^{\prime}\right)$, legs and piles $\left(K_{l p}^{\prime \prime}\right)$ are taken as:

$$
\begin{aligned}
& K_{b}^{\prime}=\sum_{l p} \frac{E A}{L} \cos ^{2} \beta_{i} \\
& K_{l=1}^{\prime \prime}=\sum_{\text {legs }}^{n_{j}} \frac{6 E I}{L_{j}^{3}}
\end{aligned}
$$

where, $\beta_{i}$ and $E A / L$ indicate the angle between brace and the axis of the horizontal member and the axial stiffness of each individual brace, respectively. The overall structural mass of each roof is computed and placed as lumped nodal masses at horizontal elevations. As the dynamic analysis of offshore jacket structure is very complex, checking and evaluating the response and behavior of these structures under dynamic loadings by making a SPM is vital, as shown in Fig.1. The steel deck is very stiff and the flexibility of the jacket structure in horizontal motion is provided entirely by the columns. An important practical aspect occurs when the platform deck alters during various normal operating conditions. For example, increasing the jacket deck mass by storing the oil in a drilling platform. This effect can change the structural dynamic properties which requires an idealized model as a concentrated mass $M$ including all topside, structural members, personnel/equipment, and stored oil masses. The added mass per unit length for cylindrical members is taken as:

$m_{\text {add }}=\pi r^{2} \rho_{\omega}(\sin \psi)$

where $\rho_{\omega}, r$ and $\psi$ are the density of the fluid, the radius of the member, and the angle between the cylindrical axis and direction of translation, respectively. More detailed information can be found elsewhere $[12,20]$.

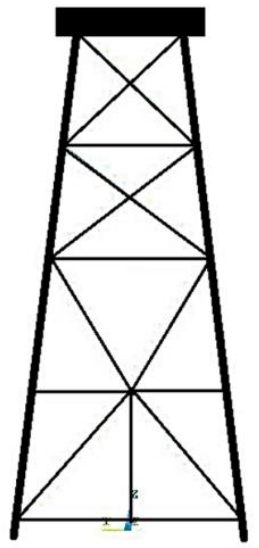

Jacket Platform

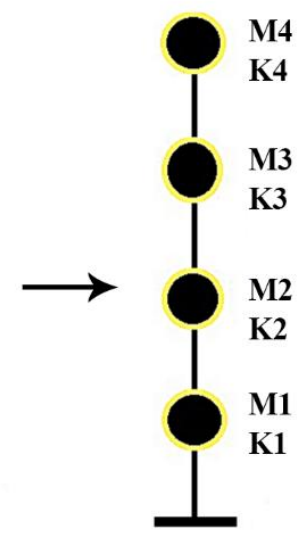

SPM Model

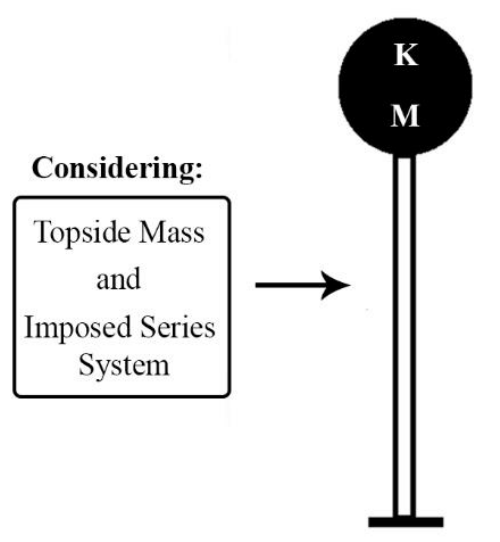

Idealized Model

Fig.1. The SPM and the Idealized Model of the Jacket Platform

Based on the FE model, which is done by the ANSYS software package, the equation of motion of the jacket platform system can be given as follows:

$$
M \ddot{X}(t)+C \dot{X}(t)+K X(t)=f(t)
$$


where $M, C$ and $K$ are mass, damping and stiffness matrices, respectively; $X(t), \dot{X}(t)$ and $\ddot{X}(t)$ denote the displacement, velocity and acceleration vectors.

\section{Experimental Test}

In this study a physical model of the jacket platform (SPD9) in scale of 1:100 is built and tested both in air and water. The model is built using the prototype dimensions by Acrylonitrile Butadiene Styrene (ABS) hollow tubes. To approach the scaled model, the dimensionless Froude numbers is taken into account based on required scaling factors. More details about the physical similitude conditions necessary for modeling an offshore structure have been presented in [21]. Density, elastic modulus, and Poisson's ratio of the experimental model material, are chosen as $1024.2 \mathrm{Kg} / \mathrm{m}^{3}, 3 \times 10^{9} \mathrm{~Pa}$, and 0.3 . The specification of the scaled model and prototype are listed in Table 1. A scheme of the scaled and the FE model are shown in Fig. 2. Experimental modal tests are implemented on the model using the shaker tests in Modal Analysis
Laboratory of the University of Tabriz using the scaled model. The measured responses are obtained from the shaker tests. The external excitation (based on white noise signals) is enforced by means of an electrodynamic exciter (type 4809) with a force sensor (AC20, APTech) driven by a power amplifier (model 2706), all made by Bruel \& Kjaer. The instrumentation is included two light uni-axial accelerometers (4508 $\mathrm{B} \& \mathrm{~K}$ ) in both the $\mathrm{X}$ and $\mathrm{Y}$ directions on each joint for response measurement and a load cell for measuring the excitation force. The frequency sampling of the test setup is chosen to be $32 \mathrm{KHz}$, and the frequency range is 0 to $200 \mathrm{~Hz}$. During the test, the structural responses are recorded as Frequency Response Functions (FRFs). The recorded data are sent to the ME'scope software package for processing. ME'scope contains a variety of FRF-based curve fitting methods. Modal curve fitting is the process used to obtain a set of modal parameters. A scheme of the performed modal testing is illustrated in Fig. 3.

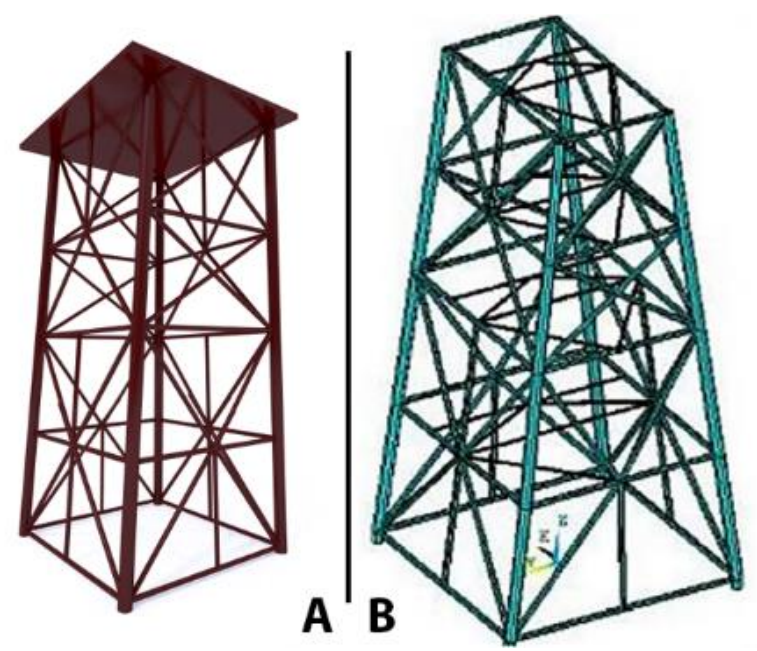

Fig. 2. The Scaled Model (A) and the FE Model (B) of the Jacket Platform

Table 1. Main Specification of the Scaled and Prototype Jacket (SPD9)

\begin{tabular}{lll}
\hline Specifications & Model & Prototype \\
\hline Height (m) & 0.74 & 74 \\
Low Level Dimensions (m) & $0.36 \times 0.35$ & $36 \times 35$ \\
Top Level Dimensions (m) & $0.29 \times 0.16$ & $29 \times 16$ \\
Jacket Weight (KN) & 0.0155 & 15500 \\
\hline
\end{tabular}



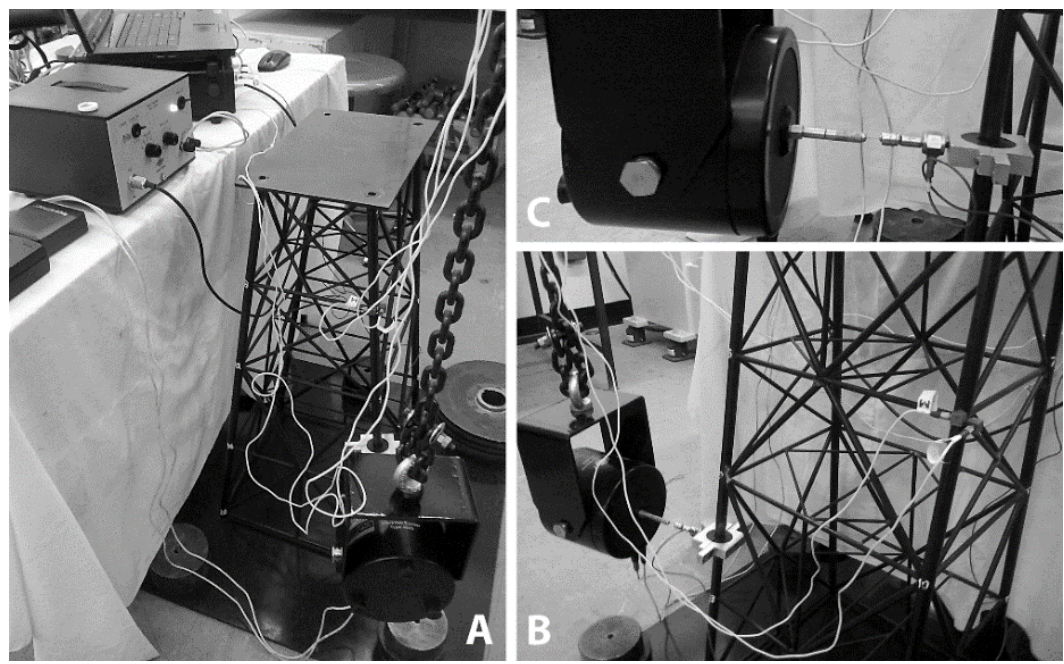

Fig. 3. Modal Testing Process Layout: (A) Overall Picture of the Experiment;

(B) Vibrator and Accelerometer Locations; (C) A Close View of the Vibrator

Model updating process is utilized with the only measured eigenvalues in this study. In this regard, the modal assurance criterion (MAC), known as mode shape correlation coefficient, between analytical mode $\Phi_{i}$ and experimental mode $\Phi_{j}$ is considered which can be defined as follow:

$$
\operatorname{MAC}\left(\Phi_{i}, \Phi_{j}\right)=\frac{\left|\Phi_{i}^{T} \Phi_{j}\right|^{2}}{\left(\Phi_{i}^{T} \Phi_{i}\right)\left(\Phi_{j}^{T} \Phi_{j}\right)}
$$

A $M A C$ value close to 1 denotes a well correlation between the two modes and a value close to 0 indicates uncorrelated ones.

\section{Results and Discussion}

In this study, an offshore jacket platform is investigated both experimentally and numerically. Updating the numerical model based on natural frequency can be defined as one of the objectives of this study. For this purpose, the natural frequencies are measured experimentally. The main problem which is shown up through measuring procedure is determining the first frequency from the recorded signals. Whereas, transmission of mechanical waves through metallic materials is easier to detect compared with transmission in polymeric materials, the instrumentations are more out in parallel with an adjustment through an integrated theoreticalnumerical-experimental analysis, which has been discussed in literature [22]. For computation of the first frequency of the experimental model both in air and water, the sudden relaxation method is implemented. The second and third mode shapes and frequencies of the numerical and experimental modal analysis are illustrated in Fig. 4. The first four experimental natural frequencies and MAC value are also listed in Table 2. The frequency of the vibration and the peak values of the different cycles are acquired by the raw curve fitting procedure. According to Table 2, there is a perfect correlation between the numerical modal and experimental modal vector.
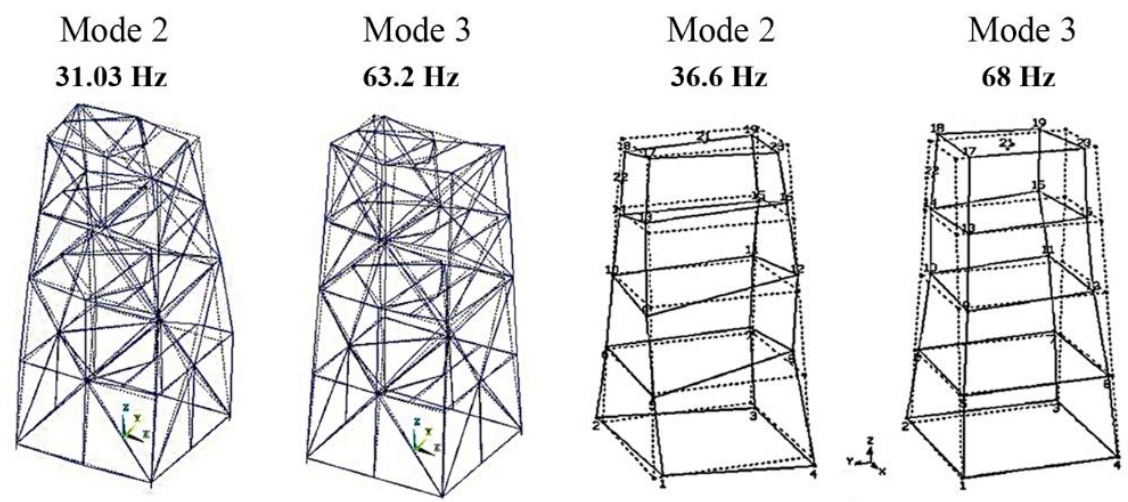

Fig. 4. The Second and the Third Mode Shapes: (A) Numerical and (B) Experimental

Table 2. Model Updating and the First Four Natural Frequencies.

\begin{tabular}{lcccc}
\hline Mode No. & Analytical & Experimental & Updated Model & MAC \\
\hline 1 & 18.64 & 11.3 & 11.18 & 0.995 \\
2 & 31.03 & 36.6 & 35.9 & 0.992 \\
3 & 63.21 & 68 & 68.91 & 0.994 \\
4 & 95.82 & 97.1 & 96.7 & 0.991 \\
\hline
\end{tabular}


Table 3. Estimated Dynamic Characteristic

\begin{tabular}{|c|c|c|c|c|c|c|c|c|c|c|}
\hline \multicolumn{9}{|l|}{ SPM } & \multicolumn{2}{|c|}{ Idealized Model } \\
\hline Element & $M_{1}$ & $M_{2}$ & $M_{3}$ & $M_{4}$ & $K_{1}$ & $K_{2}$ & $K_{3}$ & $K_{4}$ & $M$ & $K$ \\
\hline $\begin{array}{l}\text { Element } \\
\text { Values }\end{array}$ & $\begin{array}{c}5.5 \\
(K g)\end{array}$ & $\begin{array}{c}4 \\
(K g)\end{array}$ & $\begin{array}{c}3.3 \\
(K g)\end{array}$ & $\begin{array}{c}2.5 \\
(K g)\end{array}$ & $\begin{array}{c}280 \\
(K N / m)\end{array}$ & $\begin{array}{c}210 \\
(K N / m)\end{array}$ & $\begin{array}{c}170 \\
(K N / m)\end{array}$ & $\begin{array}{c}120 \\
(K N / m)\end{array}$ & $\begin{array}{c}90 \\
(K g)\end{array}$ & $\begin{array}{c}780 \\
(K N / m)\end{array}$ \\
\hline
\end{tabular}

Dynamic properties of the SPM are estimated by considering $A$ (Thickness and Diameter) and $L$ (Length) parameters. Eventually, by using the proposed method, a full agreement between the dynamic characteristics in both SPM and updated model is gained as listed in Table 3. Since the natural frequencies of the SPM and idealized model are in a good agreement with the experimental model results, the structural response under dynamic loading have an acceptable result by using the simplified procedure.

In this paper, the effects of marine growth and corrosion are perceived by considering different mass, stiffness, and initial conditions. In Fig.5A, the initial displacement of each DOF is specified as 1. A little evidence of the first mode can be observed in the fourth mode of vibration, which is due to the difference in the mass of each DOFs. The system response is redrafted by removing an initial displacement from the third and fourth DOFs in Fig.5B. The result of mass and stiffness increment in the system is illustrated in Fig.6. In comparison to Fig.5A, the curves of Fig.6A have lower frequencies and higher spreading outs.
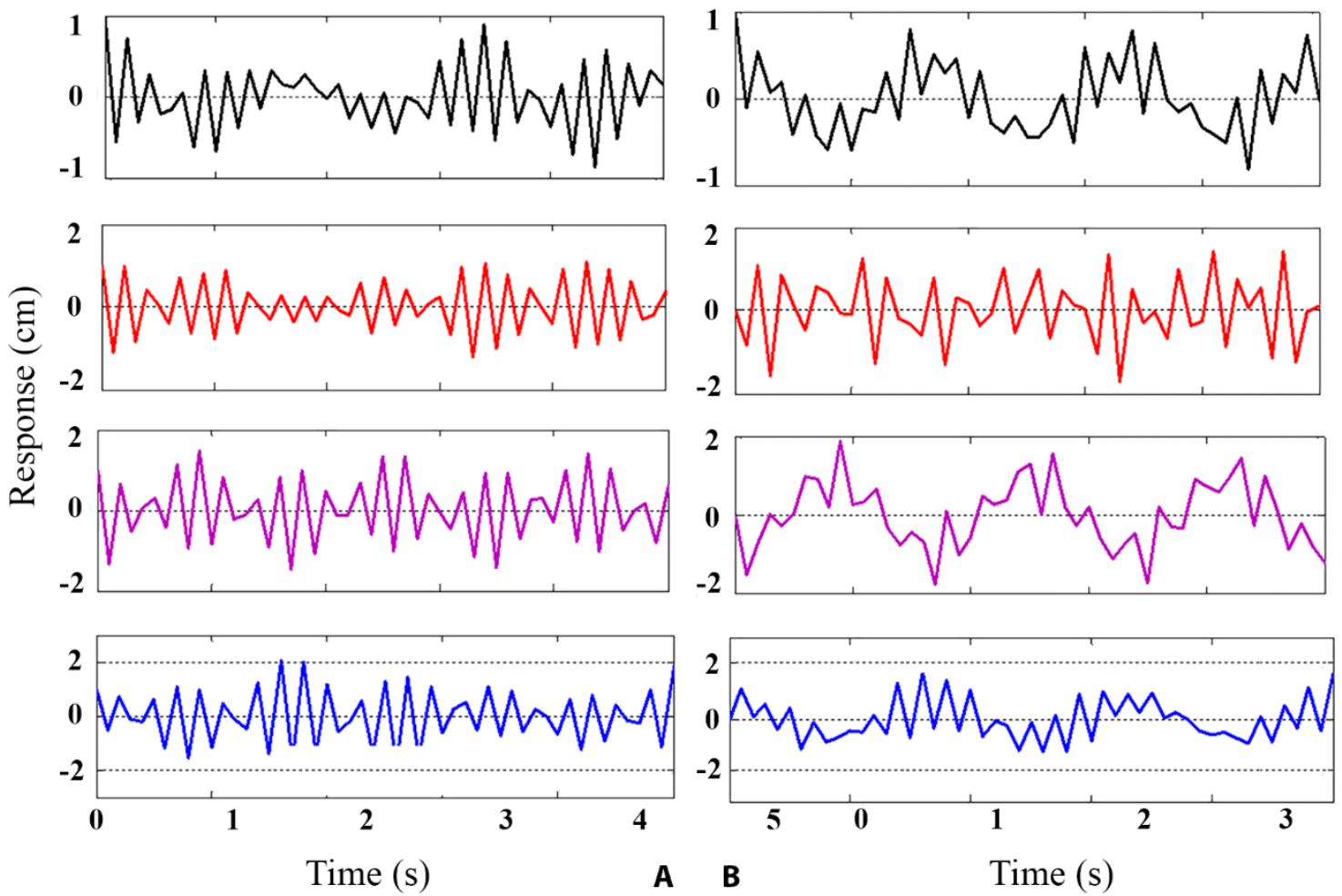

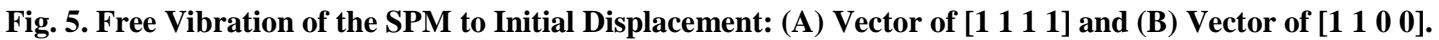



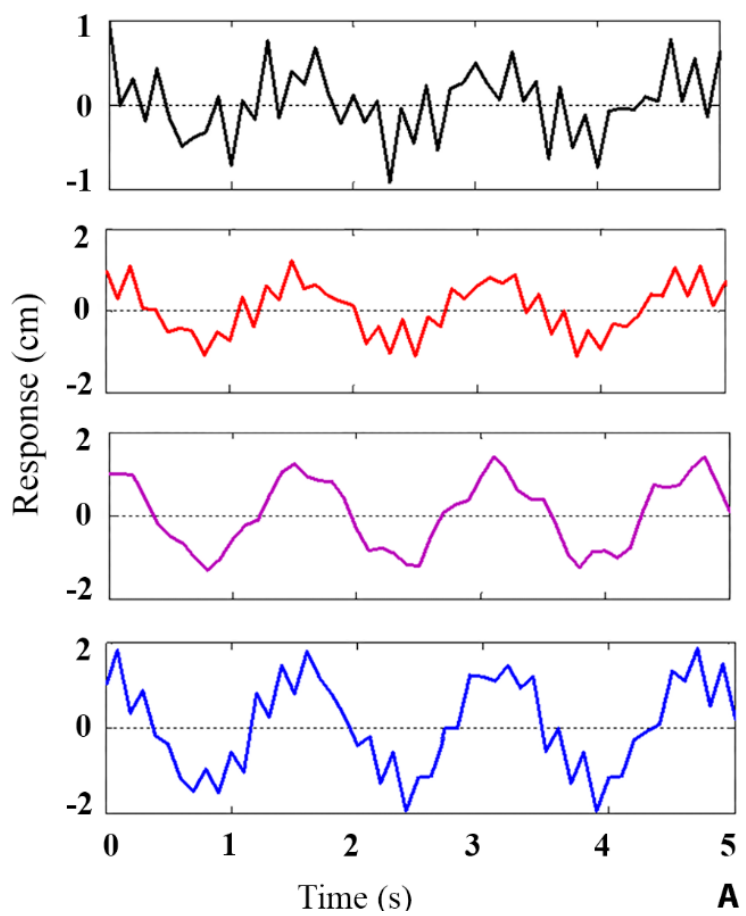
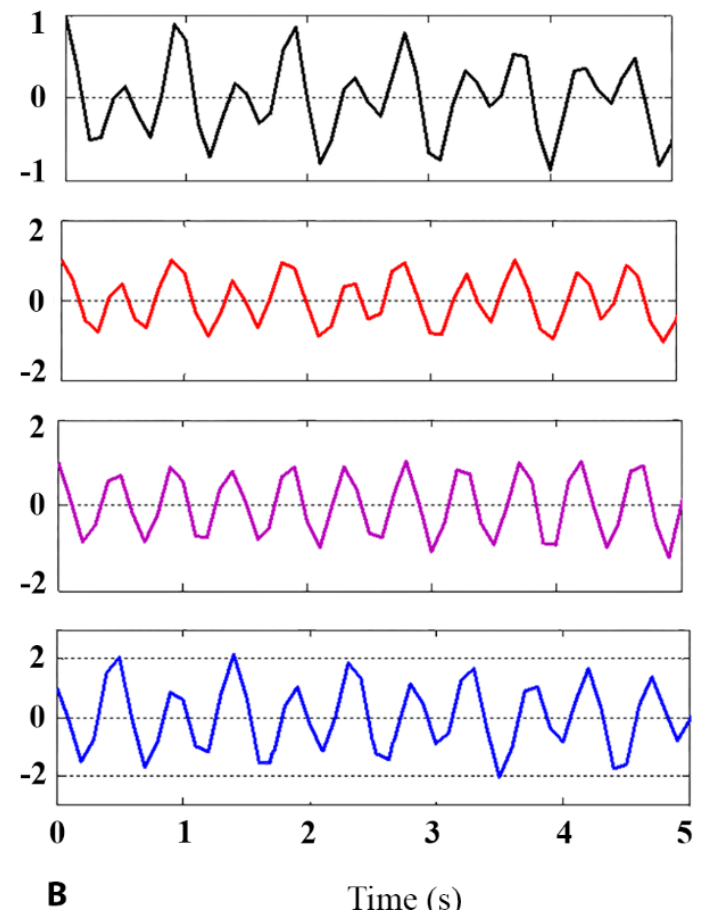

Fig.6. Free Vibration of the SPM with: (A) Mass Increment (Effects of marine growth) and (B) Stiffness Reduction (Effects of corrosion)

For studying the variation of vibrational characteristics after adding the deck weight and considering constant stiffness, two parameters as initial velocity changes and initial displacement changes are investigated. It should be noted that the frequency decreases as the mass of the system increases. It is evident that the phase angle and maximum amplitude are also functions of the natural frequency. As it is shown in Fig.7, the maximum amplitude decreases with deck weight increment because of the corresponding reduction in natural frequency. As a result, the phase shift diminishes as the peak of vacillation gets closer to $t=0$. The response of the system is also investigated with different magnitude of force and natural frequencies. According to Fig.8, it is obvious that the magnitude of the response is directly a proportion of the external force. It is also seen that as the oscillatory motion begins, the response is not centered on zero. However, the value of center point depends on the magnitude of the external force. In Fig.9, the variation of natural frequency is illustrated. It is shown that two variations in the response is existed. First, the rate of response which decreases exponentially (the effect of damping) is increased; it means that the response is stabilized more quickly. Second, the oscillation frequency decreases as the natural frequency increases. This phenomenon can be explained due to damped frequency increment as the natural frequency increases.

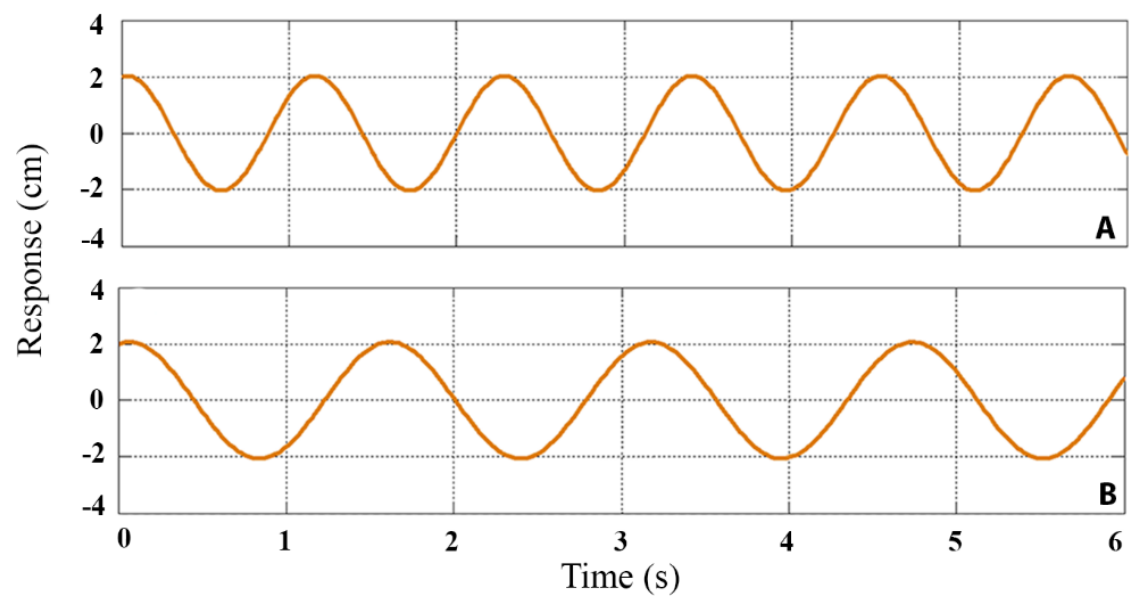

Fig. 7. Simple harmonic motion of the idealized model: (A) Before and (B) After considering the added deck weight. 

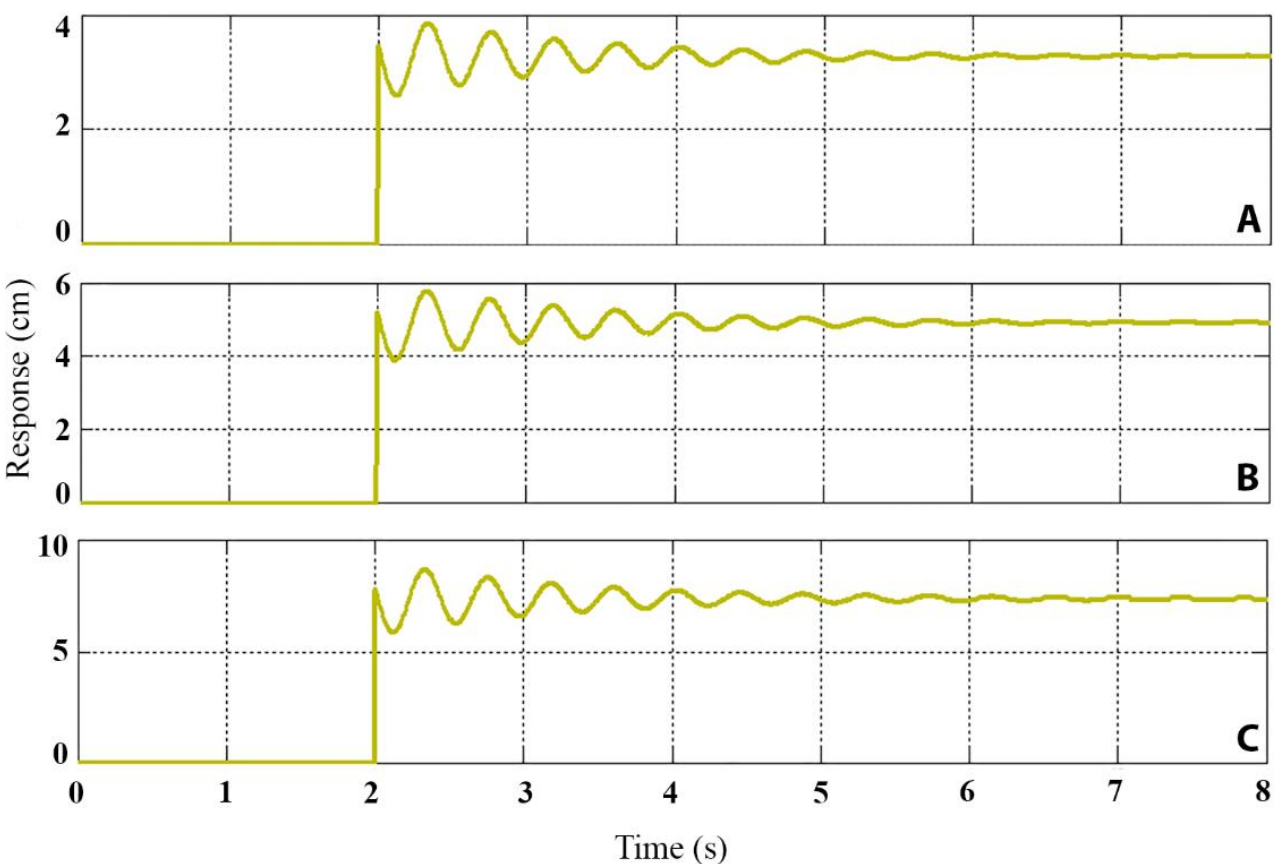

Fig.8. Structural responses of the idealized model due to rectangular impulse by considering different force magnitudes (A) 5; (B) 10; and $(C) 15$.

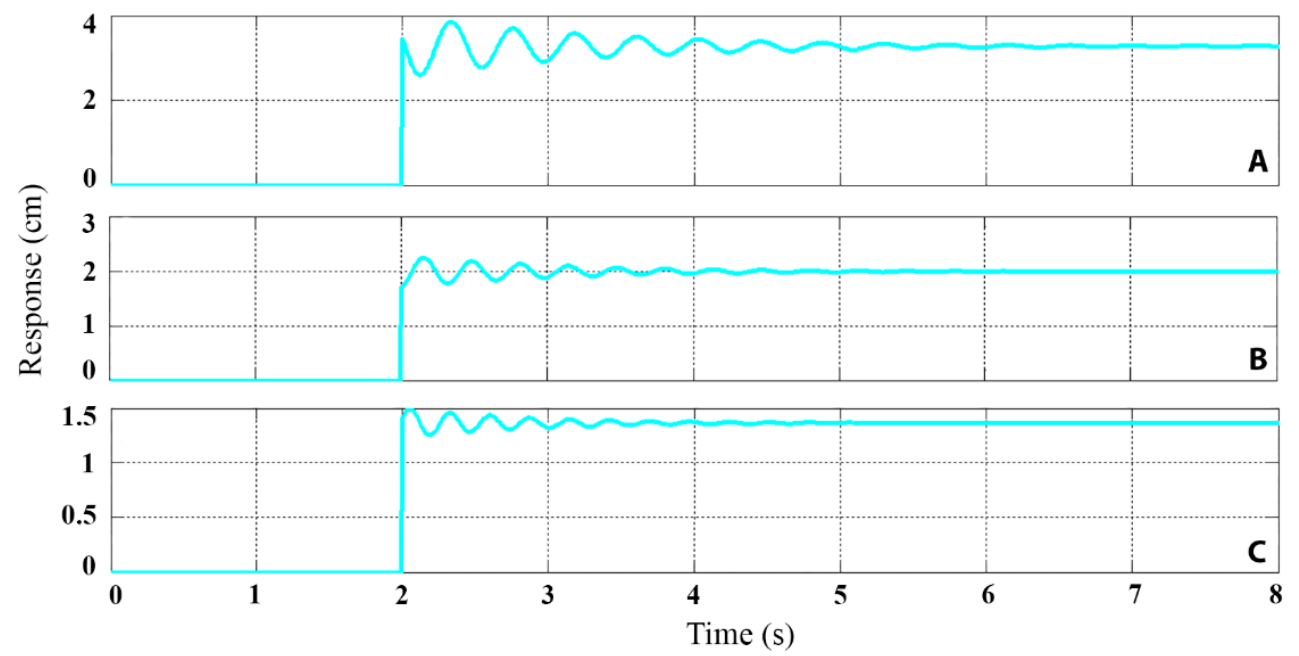

Fig.9. Structural responses of the idealized model due to rectangular impulse by considering different natural frequencies: (A)14.42 $\mathrm{Hz}$; (B)20 Hz; and (C)25 Hz.

Seismic response of the jacket platform is studied through the idealized model under El Centro earthquake acceleration for detailed investigation of the system response. The effect of added deck weight on the behavior of the platform model is evaluated by using the Newmark- $\beta$ technique [23]. The time history of acceleration, displacement and bending moment of the jacket platform are presented in Figs 10, 11, and 12, respectively. The values of bending moment are gained through the idealized model based on the equivalent mass and stiffness parameters $(M, K)$, as presented in Table 3. The results demonstrate that the maximum displacement response and the values of bending moment at the base of model are higher in comparison with the model before considering added deck weight. Maximum peak responses of the ideal model with and without adding the deck weight are listed in Table 4. 

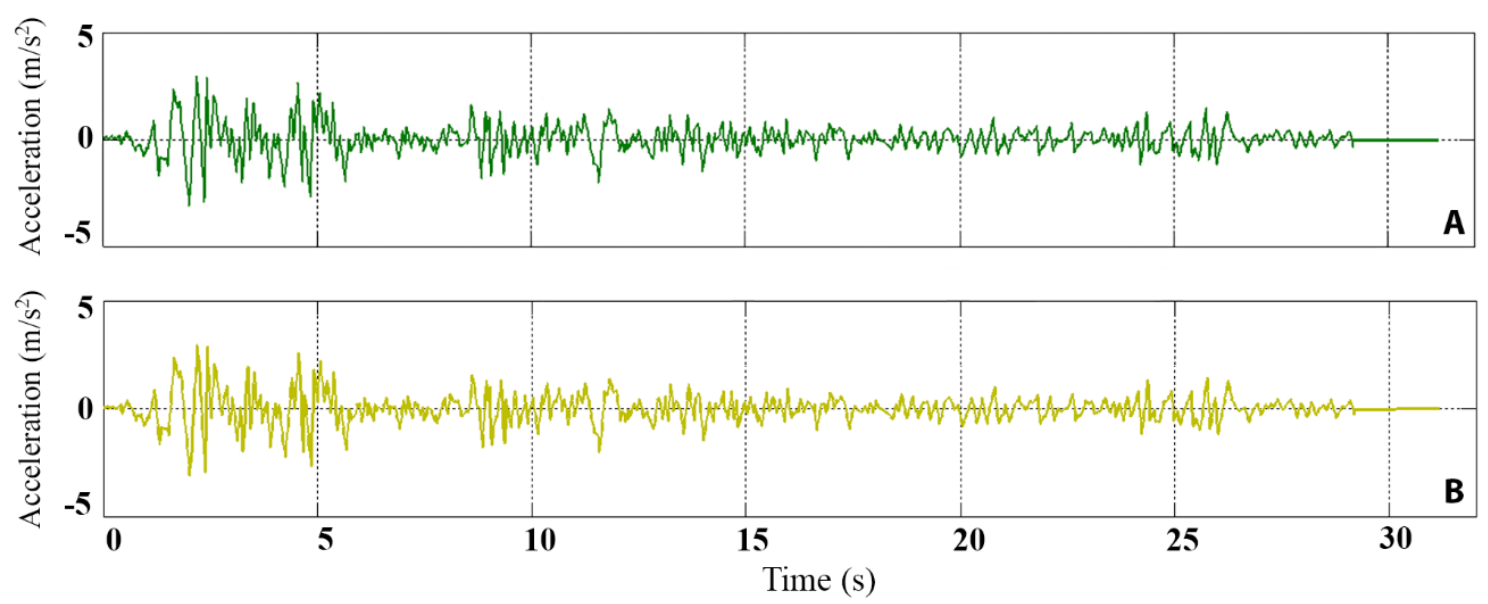

Fig. 10. Acceleration Time history of the Jacket Response under El Centro Excitation: (A) Before and (B) After Considering Added Deck Weight
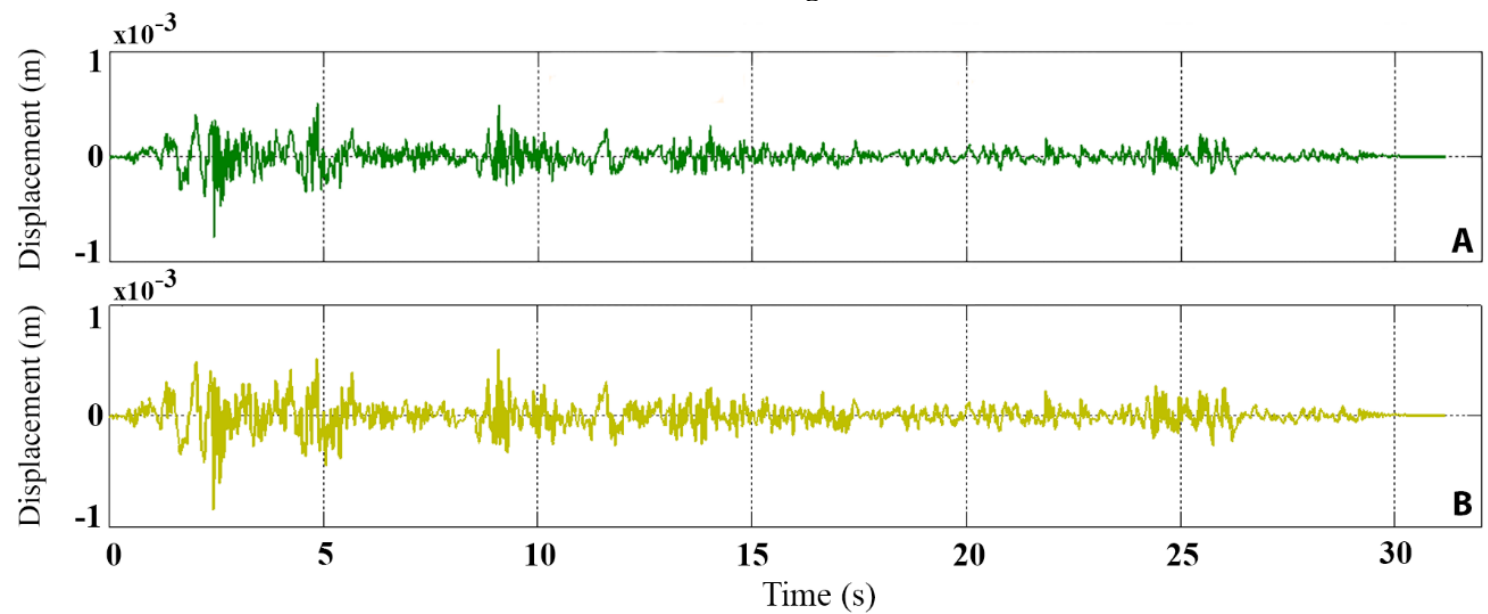

Fig. 11. Displacement Time history of the Jacket Response under El Centro Excitation: (A) Before and (B) After Considering Added Deck Weight
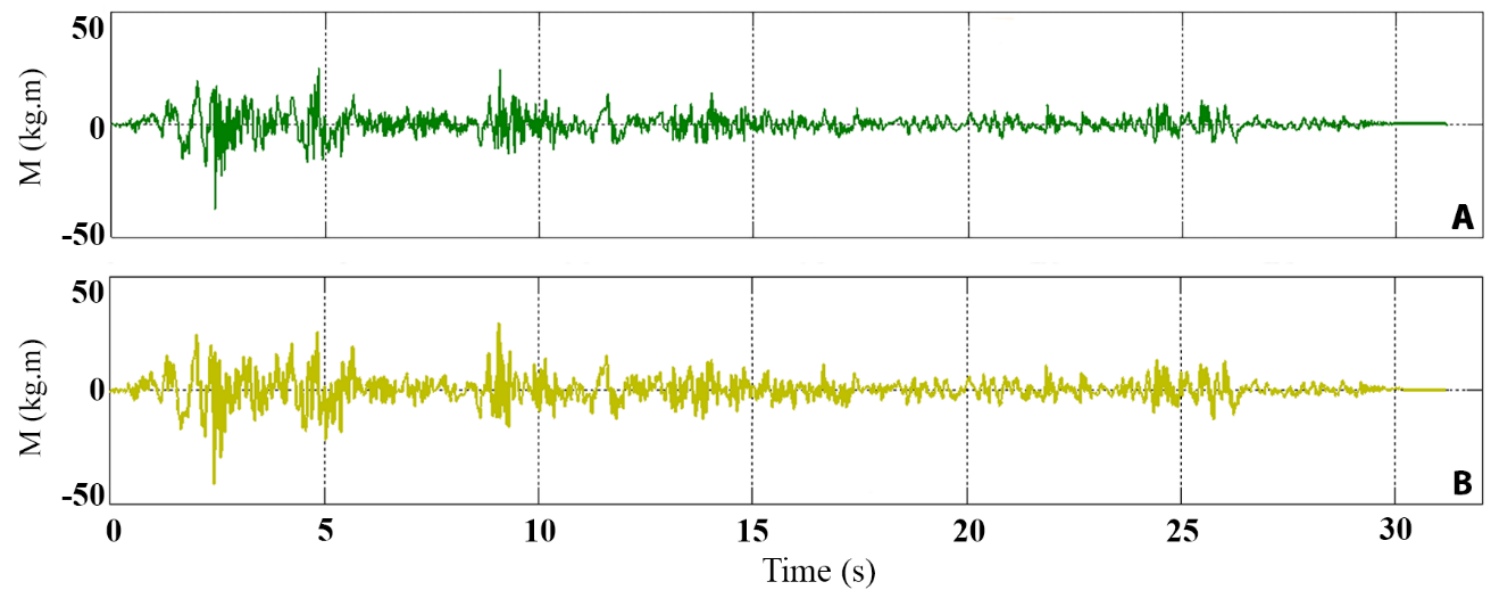

Fig. 12. Bending Moment Time history of the Jacket Response under El Centro Excitation: (A) Before and (B) After Considering Added Deck Weight

According to the results of acceleration time history (Fig. 10), it is seen that a good agreement is existed between the structural behavior and dynamic response of the jacket in real and the simplified models. Based on the results of Fig.11 and 12, the whole response of the system increased slightly which is obviously due to deck weight increment in the jacket platform. As a conclusion, it can be noted that the proposed method can be used for simplification of calculation process, bias error reduction and time and cost reduction procedure in significant research projects due to the efficient behavior and response of the simplified models. 
Table 4. Maximum Peak Response of the Jacket Before and After Considering Added Deck Weight

\begin{tabular}{lcc}
\hline Specification & Before & After \\
\hline Acceleration $\left(\mathrm{m} / \mathrm{s}^{2}\right)$ & 2.927 & 2.972 \\
Displacement $(\mathrm{mm})$ & 0.502 & 0.595 \\
Bending Moment $(\mathrm{Kg} . \mathrm{m})$ & 24.488 & 29.031 \\
\hline
\end{tabular}

\section{Conclusions}

In this paper, a scaled hydro-elastic model of a jacket platform (SPD9) is utilized for the experimental evaluation of a simplified approach by using improved simple dynamic model. The uncertainty effects on the results are also considered as a perspective of the study for assessment of the dynamic behavior of jacket structures. Model reduction technique is applied in model updating process. Therefore, an improved reduction approach associated with the model updating is developed based on the static condensation scheme. The model is updated numerically based on the MAC factor after calculation of the equivalent frequencies by the FE model. A baseline FE model is improved through updating the numerical model by using the experimental data. Finally, the dynamic characteristics of the improved SPM and idealized model are determined according to the updated model properties. The effects of marine growth, deck weight increment and corrosion are also investigated. Based on the results, model weight (marine growth) or deck weight increment causes the displacement response to decrease under free vibration loading and to increase under proposed earthquake excitation. Also, the results indicate that the displacement response increases as the stiffness of the SPM decreases (effect of corrosion), during the free vibration. Also, adding more deck weights to the system causes the response of the system to increase under earthquake excitation. According to the results, an empirical verification of a simple dynamic model of an offshore jacket structure leads to a reduction in amount of calculations and expenses for this case study.

\section{References}

1- Hokmabady, H., Mojtahedi, A., Lotfollahi Yaghin, M., Farajpour, I. 2019. Calibration and Bias-Correction of the Steel Offshore Jacket Platform Models Using Experimental Data. J Waterway Port Coast and Ocean Eng. 145(3):04019008-1-15. https://doi.org/10.1061/(ASCE)WW.19435460.0000509.

2- Ewins D.J. 2000. Model testing: theory, practice and application. second ed. Research studies press.

3- Baruch M. and Wis M. 1978. Optimization procedure to correct stiffness and flexibility matrices using vibration tests. The Am. Ins. Aero. And Astro. 16(11):1208-1210.

4- Denoyer K. K. and Peterson L. D. 1997. Method for structural model update using dynamically measured static flexibility matrices. The Am. Ins. Aero. And Astro. 35(2):362-368.

5- Lin, R. -M., Lim, M. -K. and Du, H.,"Improved inverse eigensensitivity method for structural analytical model updating," Trans. ASME, Vol. 117, pp.192-198, 1995.

6- Guyan, R.J. 1965. Reduction of stiffness and mass matrices. The Am. Ins. Aero. And Astro. 3:380-392.

7- O'Callahan J. 1989. A procedure for improved reduced system (IRS) Model. 7th IMAC. Las Vegas. USA.

8- Zeinoddini, M., Matin Nikoo, H., Estekanchi, H., 2012. Endurance Wave Analysis (EWA) and its application for assessment of offshore structures under extreme waves. Applied Ocean Research 37(0): 98110.

9- Najafian G. 2007. Application of system identification techniques in efficient modelling of offshore structural response. Part I: model development. J. App. Ocean Res. 29:1-16.

10- API-RP2A. 2000. Recommended practice for planning designing and constructing fixed offshore platform-working stress design. 21st ed. Washington, DC: American Petroleum Institute.

11- Zeinoddini M. 2006. Design and performance of fixed offshore platform. Iranian National Oceanology Institute. Persian.

12- Asgarian B, Lesani M. 2009. Pile-soil-structure interaction in pushover analysis of jacket offshore platforms using fiber elements. J. Cons. Steel Res. 6:209-18.

13- Gomathinayagam S, Vendhan CP, Shanmugasundaram J. Dynamic effects of wind loads on offshore deck structures - a critical evaluation of provisions and practices. J. Wind Eng. and Ind. Aerodyn. 84(3):345-67.

14- Winsor F. 2003. Evaluation of methods to remove inertial force from measured model wave impact force signals. Ocean Eng. 30(1):47-84.

15- Elshafey, A.A, Haddara, M.R, Marzouk, H. 2009. Dynamic response of offshore jacket structures under random loads, Marine Struc. 22:504-521.

16- Bargi, K., Hosseini, S., Tadayon, M., Sharifian, H., 2011. Seismic response of a typical fixed jacket-type offshore platform (SPD1) under sea waves. Open J. Mar. Sci. 1:36-42.

17- Park, M., Koo, W., Kawano, K. 2011. Dynamic response analysis of an offshore platform due to seismic motions. Eng. Struct. 33:1607-1616.

18- Hutton, D.V. 2004. Fundamentals of Finite Element Analysis. McGraw-Hill. New York. 
19- Bea, R.G., Stear J.D., 1998. Simplified strengthlevel earthquake assessment of jacket-type platforms, Eighth Int. Offshore and Polar Engineering Conference, Montreal, Canada.

20- Zhou, B., Han, X., Tan, S.K, 2014. A simplified computational method for random seismic responses of a jacket platform. Ocean Eng. 82:85-90.

21- Chakrabarti, S. K. 1994. Offshore structure modeling. World Scientific Publishing Co. Pte. Ltd. Singapore.
22- Hosseinlou, F., Mojtahedi, A. 2016. Developing a robust simplified method for structural integrity monitoring of offshore jacket-type platform using recorded dynamic responses. J. App. Ocean Res. 56:107-118.

23- Chopra, A., 2005. Dynamics of Structure: Theory and Application to Earthquake Engineering, second ed. Tsinghua University Press. Beijing. China. 